\title{
Computer Network Performance Optimization Approaches based on Distributed System with the Cloud Computing Environment
}

\author{
Song Jiajia \\ ${ }^{1}$ Henan Univ. of Science and Technology, Luo Yang 471000, China
}

\begin{abstract}
In this paper, we conduct research on the computer network performance optimization approaches based on distributed system with the cloud computing environment. The information age, network is everywhere, increased, but the network speed is not enough. Blame the reason, on the one hand, to speed up the network construction, on the other hand, in actual use and construction should pay more attention to improve the performance of network problems. Computer network performance problem is very complex and important, as hundreds of thousands of the computers connected together, often appear unable to predict the result of complex interactions, this complexity to cause a decline in network performance and often difficult to determine why. There are very few in the actual use of basic theory of or system as this article explores many issues related to the network performance, so as to comprehensively and systematically understands what performance problems in computer network and how to solve the performance problems and from their own experience gained from practical experience, some real examples, points out the key of the problem. Further research plan and schedule are also discussed with final conclusion in the final part.
\end{abstract}

Keywords: Computer Network, Performance Optimization, Cloud Computing, Distributed System, General Approaches.

\section{Introduction}

Today's information society will become a huge number and the information circulation highly developed information processing society. For the large-scale, many features of information, we should not only meet all kinds of high speed, efficient computing ability, more to be processed information in high speed, high performance transmission ability of the communication that could be reflected from the listed parts. (1) Any system contains control. There is a widespread orderly and disorderly open system and the transformation rules. All parts of the system as a whole in the coordination and synchronization, and the correlation between the whole and the environment that is considered as a whole since the conditions of the organization and if some components within a system discrete, friction, with elbow each other, cannot be effectively coordinated, so the system was in disorder, not well play the overall function, or even collapse, collapse. (2) Computer network was mainly used for electronic computers in variety of general network equipment and communication resources, under the influence of all kinds of the intelligent control of large and complex system and it is a high efficient production, processing, distribution and control the material basis for the basic information. (3) Information theory system theory and cybernetics are research information, systems and control problem of transection science. Information theory mainly studies the nature of the information, research how to use mathematical theory to describe and measure method of information, as well as the basic principle of transmission, as well as the processing information [1-2].

Computer network is information network system, to protect the safety of computer network as the essence is the safety control of network information. Study information network safety control, must be the organic combination of system theory, cybernetics and basic information theory, from the viewpoint of the system theory, information network security control system from the general angle of cybernetics research information network safety primary control structure, service, mechanism and technology, describe information network security from the perspective of the information theory and the control efficiency. In the later sections, we will propose new computer network performance optimization approaches based on basic distributed system with the cloud computing environment and the related theories.

\section{Cloud Computing Environment}

Cloud computing service level is set depending on the type of service is service, with the computer network architecture in different levels of classification. Each level in the computer network to achieve a certain function, the certain correlation between layer and layer and the level in the cloud computing architecture can be divided, namely a hierarchy that can be individually complete a user request without the need for the other levels to provide the necessary services and support.

The emergence of cloud computing represents the computing power can also serve as a kind of goods through the Internet transmissions and general distribution, service providers will calculation, services and applications, as a kind of public facilities provided to the core user. Cloud computing has the ability to do this that is composed of the following a few core characteristics. (1) Users can use cloud computing services anytime and anywhere through the network to provide all kinds of computing resources and capabilities, in the aspect of infrastructure, platform, level, and the application of the specific level for personalized service. (2) Through the cloud services, users according to need to get some of relate virtual resources and computing power, rather than buying, save and maintain specific IT hardware or the software products. (3) When users in the use of the service is not need to know the 


\section{International Journal of Science and Research (IJSR) \\ ISSN (Online): 2319-7064}

Index Copernicus Value (2013): 6.14 | Impact Factor (2014): 5.611

specific architecture of the cloud internal resources, service mode and geographic position, only need to interact with the service providers can access to general resources. In cloud computing, management process and calculation process are finished on the logic of virtual computing resources, without considering the difference between the physical resources environment in practice, more easy to management and the distribution. Its function is to can use the Internet to form an open platform for users, users can according to their own needs, in this platform of basic cloud computing provides mathematical calculations or on your own data information for conservation and utilization of operation [3].

\section{The Distributed System}

At present commonly used way to share data is set up a centralized database in the computer network, each node can access the database through a computer network, there are many shortcomings but the way such as large communication overhead, such as poor performance, usability, and difficult to manage. If in accordance with the requirements of data source and the scattered reasonable distribution in system, establish multiple databases in lieu of a centralized database, most of the data can be on-site access, and other resources in the database can be shared at the same time. Obviously this is more than just create a centralized primary database is more reasonable. Due to the distributed management has logical unity, is a whole different data to the user, the user need not care about data stored in where, where processing. On the contrary, the centralized management of data, to take in the central processor or storage device failure occurs when the risk of all visitors will be in trouble [4-5].

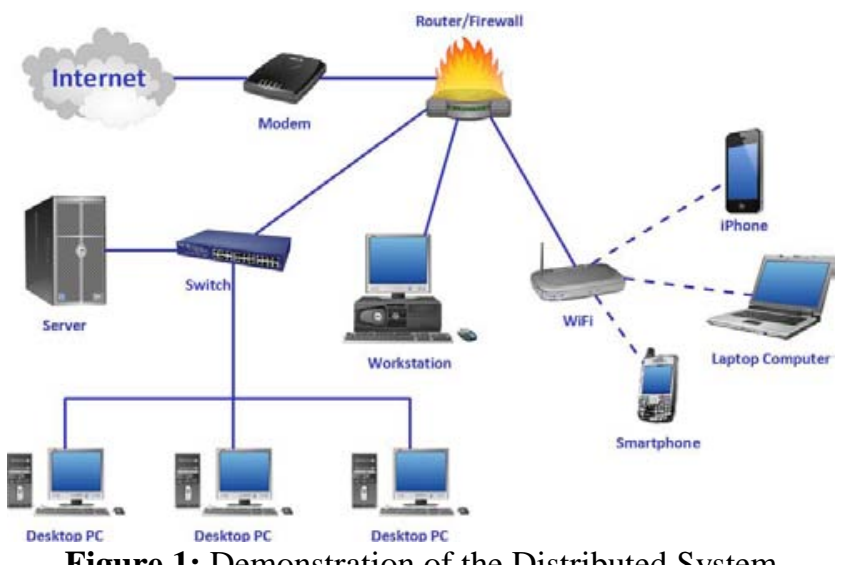

Figure 1: Demonstration of the Distributed System

A distributed system is a kind of multiple processors. Each processor constitutes unified system via the Internet. System structure of distributed computing, namely the original system within the CPU processing task processor that can spread to the appropriate implement different functions of each processor coordinate with each other, while sharing the peripherals and software of the system. Compared with the centralized system, the advantages of the distributed system mainly reflect in the following. (1) With a strong scalability. Whether can scale easily, is closely related to the stand or fall of performance, to shrink and the expansion of a distributed system, high flexibility and extensibility. (2) The system has the ability to respond quickly. Distributed systems will be accepted by any of task assigned to all hosts dominated the parallel execution, so its response time is shortened greatly.

\section{Network Performance Optimization}

According to the specific analysis of the computer network, from physical network optimization, equipment optimization and safety optimization and detail optimization, technical optimization and so on aspects to discuss implementation of network optimization. We can adopt the method of network structure optimization and transmission medium optimization solution. The structure optimization is to minimize the node together, directly within the building set convergence layer, from the core to gather all the direct logic connection, the original in the middle of the active node is omitted. Network equipment optimization and this is according to the different network applications and network flow analysis based on his own decision update network equipment. On the premise of the rational utilization of the original equipment at the same time, the update software version and add new application module to optimize the network [6].

Technical aspects of the optimization are mainly from the perspectives of routing protocols, adjusted and optimized. Highly efficient routing protocol, general VLAN planning, reasonable IP geology can be divided into different extent, improve the speed and the overall performance of network. Among them the purpose of the routing optimization is to ensure that the network has good stability, scalability and fast convergence. Approach is to add less core routing entry in the routing table, to set up the routing policy and rules, which must be followed in data flow to achieve the purpose of the precise control of data flow and close the connection user network routing receiving and core routing switch pressure decomposition methods, etc.

\section{Summary and Conclusion}

In this paper, we conduct research on the computer network performance optimization approaches based on distributed system with the cloud computing environment. Computer network management system is a basic network management software system, it is network management workstation on the network activity monitor, collect various communication information within the network, and analyzes the information and make the necessary processing and other activities. Usually, the function of the computer network management mainly include: accounting management, fault management, performance management, configuration management and basic security management. In the several major management functions, the network fault management is one of the most widely implemented, it can use some of the feasible detection technology to determine the cause of the network failure and make the corresponding processing. The purpose of network management is to ensure network security, reliable and efficient operation of network resources can get reasonable allocation and effective use, so as to optimize the network performance to reduce the cost of network maintenance. Our research proposes the novel paradigm of the related issue that will enhance the general performance. 


\section{References}

[1] Tartakovsky, Alexander G., Aleksey S. Polunchenko, and Grigory Sokolov. "Efficient computer network anomaly detection by changepoint detection methods." Selected Topics in Signal Processing, IEEE Journal of 7.1 (2013): 4-11.

[2] Kizza, Joseph Migga. "Computer Network Fundamentals." Guide to Computer Network Security. Springer London, 2013. 3-41.

[3] Mishra, Bimal Kumar, and Samir Kumar Pandey. "Effect of anti-virus software on infectious nodes in computer network: a mathematical model."Physics Letters A 376.35 (2012): 2389-2393.

[4] Ellison, Nicole B., et al. "Cultivating social resources on social network sites: Facebook relationship maintenance behaviors and their role in social capital processes." Journal of Computer-Mediated Communication 19.4 (2014): 855-870.

[5] Gan, Chenquan, et al. "A propagation model of computer virus with nonlinear vaccination probability." Communications in Nonlinear Science and Numerical Simulation 19.1 (2014): 92-100.

[6] Tsay, Joe-Kai, and Stig F. Mjølsnes. "A vulnerability in the umts and lte authentication and key agreement protocols." Computer Network Security. Springer Berlin Heidelberg, 2012. 65-76. 